\title{
An Enhanced Centrifuge-Based Approach to Powder Characterization: \\ Experimental and Theoretical Determination of a Size-Dependent Effective Hamaker Constant Distribution
}

Myles C. Thomas, Stephen P. Beaudoin *

School of Chemical Engineering, Purdue University, Forney Hall of Chemical

Engineering, 480 Stadium Mall Drive, West Lafayette, IN 47907-2100, United States

${ }^{*}$ Corresponding author at: School of Chemical Engineering, Purdue University, Forney Hall of Chemical Engineering, 480 Stadium Mall Drive, West Lafayette, IN 47907-2100, United States. Tel.: +1 765494 7944; fax: +1 7654940805 .

E-mail addresses: thoma200@purdue.edu (M.C. Thomas), sbeaudoi@purdue.edu (S.P. Beaudoin).

\section{Introduction}

Solids handling issues have a profound effect on many industrial processes. In particular, the pharmaceutical and food industries produce many products that are either solids or suspensions of solids in their final form [1-8]. Uncontrolled, unpredictable particle adhesion to surfaces and/or other particles is a main source of particle processing problems such as: caking, poor flowability, dust hazards, consolidation, and segregation $[9,10]$. 
Within the pharmaceutical industry, particle adhesion affects processes such as mixing, conveying, granulation, and compaction [11]. In terms of simple contact, which does not involve mechanical interlocking or chemical bonding between the surfaces, the adhesion force can be considered to be the force needed to separate two surfaces from contact. The adhesion force is influenced by the contact made by the two surfaces, thus surface roughness of the interacting bodies significantly impacts the resulting force. The effects of surface roughness on the van der Waals adhesion force is of primary interest here $[12,13]$. Surface roughness affects adhesion because contact occurs between surface asperities, which results in great variation in the mass of material in close contact (within a few nanometers) between the two surfaces [14]. While the effect of this variation may be to either increase or decrease the adhesion, depending on the intimacy of the contact, in general the roughness leads to reduced adhesion between solid surfaces. A number of approaches have been implemented to quantify the effects of roughness on the van der Waals adhesion. These include describing asperities on surfaces using spheres [15]; using hemispheres [16-19]; using a geometrical correction that captures the effect of roughness by considering that only one hemispherical asperity on a rough particle interacts with an opposing surface [20]; using an empirical correction that reduces the classic relation between a smooth sphere and a flat plate based on the rms roughness [21]; using the particle roughness to calibrate fractal models for the surface texture [22-26]; using an effective surface energy to describe the effect of roughness in a finite element description of a classical work of adhesion model [27]; or by using a statistical model for the deformation of spherical asperities in contact between two surfaces under load [28]. While these models have all been validated 
successfully in different settings, none can use a relatively small number of measurements to effectively describe the adhesion between a population of particles (a powder) and a surface as a function of the size of the particles in the powder. A method to evaluate the effect of individual particle surface roughness on the adhesion of the ensemble can lead to better understanding and modeling of powder behavior and improved powder processing performance.

To assist with the development of accurate descriptions of powder behavior, adhesion force measurements are often extremely helpful. Various techniques are available to measure adhesion forces, including: the centrifuge technique, aerodynamic technique, hydrodynamic technique, impact-separation method, ultrasonic vibration, electrical field detachment technique, and force microscopy [11]. The type of measurement used depends on the mechanisms affecting the particle adhesion, the system properties, and the resources available. The centrifuge technique can be used to measure particle adhesion by relating the radial force induced by the rotation of a centrifuge to the adhesion force of the particles experiencing the applied force. It is a practical method that measures particle/powder adhesion, is simple to perform, and describes the adhesion behavior of an ensemble of particles in terms of the average adhesion force [29-33]. While knowing the average adhesion force for a powder is useful, knowledge of the adhesion force distribution would be far more useful, especially if this distribution could be correlated with easily measured particle/powder properties. The goal of this work is to demonstrate that the analysis of the classical centrifuge technique can be modified to provide this higher quality information about particles/powders. Previous work established the theoretical basis for this current 
study, which includes experimental results and simulations with the modified analysis [34]. For this purpose, experiments using the centrifuge technique were performed with silica particles adhering to stainless steel. A simulated powder with the same size distribution as the silica used experimentally was developed. This powder was assumed to be comprised of smooth spheres. The removal of this simulated powder from the centrifuge plates was calculated using the classical van der Waals model for adhesion between a non-deformable sphere and a flat plate. An effective Hamaker constant distribution was applied to force agreement between the calculated and observed powder adhesion. This distribution captured the effects of any shape and topographical nonuniformities within the real powder on the van der Waals adhesion. In this manner, a powder consisting of non-ideal particles is characterized in terms of an ideal system with a fitted Hamaker constant distribution and no loss of accuracy in the description of the adhesion forces.

\section{Materials and Methods}

\subsection{Materials}

The centrifuge method of particle adhesion measurement was performed with silica particles on stainless steel centrifuge plates to characterize the particle adhesion based on the way the particles were removed from the substrate. Colloidal silica particles (AEROPERL® 300 Pharma) were supplied by Evonik (U.S.A.). These particles were used in this study because they are nominally spherical and rigid. The particles have a density of $2.2 \mathrm{~g} / \mathrm{cm}^{3}$. Stainless steel (type 316, McMaster-Carr Supply Company, 
9745K15) was used as the substrate because of its prevalence in powder processing operations in many industries that perform solids handling operations. The steel was cut into $1 \mathrm{~cm} \mathrm{X} 1 \mathrm{~cm}$ square coupons that fit into custom-modified centrifuge tubes.

\subsection{Methods}

\subsubsection{Centrifuge Technique}

The centrifuge technique is a common method that has been used since the early 1960s to measure powder adhesion [29-33]. Its ability to provide a mean adhesion force for a powder, coupled with its ability to measure the adhesion of particles on different surfaces in a single experiment, makes it a valuable tool. A description of the centrifuge method can be found elsewhere [32]. By way of review, the centrifuge process works as follows:

1. Particles are dispersed on plates that will be mounted in the centrifuge tubes.

2. An optical image of the plates is obtained and any number of image analysis software packages is used to align images and count the number of particles on the plates (ImageJ from NIH was used in this case) [35-37].

3. The plates are mounted in a holder that is placed in a specially designed centrifuge tube. In this manner, the plates are placed so that the particles face outward and each plate is parallel to the axis of rotation of the centrifuge.

4. The centrifuge is rotated at a chosen speed for a fixed time.

5. The plates are removed and the particle counting process is repeated.

6. The plates are remounted in the centrifuge tubes. 
7. The centrifuge is rotated again, for the same amount of time but at a higher rotational speed.

8. Steps 5-7 are repeated until the working range of centrifuge speeds is reached or until all particles are removed.

The basis of the method is that detachment of a particle adhered to a surface that is parallel to the axis of rotation of the centrifuge will occur when the adhesion force is exceeded by the radial force resulting from the centrifuge rotation. This radial force, $F_{c e n t}$, is expressed in terms of $m$, the mass of the particle; $\omega$, the angular velocity; and $r_{c}$, the distance from the center of rotation, by:

$$
F_{\text {cent }}=m \omega^{2} r_{c}
$$

It is important to remember that this only holds true if the radial force is acting perpendicular to the surface on which the particle is adhered. A modified centrifuge tube which holds the substrate parallel to the axis of rotation is used for this purpose. The apparatus and method of the centrifuge technique is illustrated in Fig. 1. The system used in this work is a fixed angle $\left(34^{\circ}\right)$ rotor that holds the centrifuge tube, substrate holder, and substrate stationary within the rotor. In this manner, the radial force is normal to the substrate and is countering the adhesion force of the particles on the substrate when the centrifuge is in operation.

The centrifuge technique was carried out by using silica particles on stainless steel substrates. The silica particles were dispersed onto the stainless steel by aerating the particles with compressed gas in a covered container and allowing the particles to land on the substrate. In this manner, a large number of particles could be placed on the 
substrate while avoiding particle agglomeration. Optical images of the deposited particles were captured using a Nikon SMZ1500 Optical Microscope. The image taken prior to centrifugation is the reference image containing the total number of particles on the substrates. This image will be referred to as the initial image or the 0 RPM image. An Eppendorf 5804 fixed-angle rotor centrifuge was used for the study. Rotational speeds of $1500,3000,4500,6000,7500,9000$, and 10500 RPM were applied for oneminute intervals to perform all experiments. After each experiment at each rotational speed, an image was captured using the optical microscope. Upon completion of each experiment, the captured images were processed using ImageJ to determine the number of particles remaining as a function of the rotational speed. It is important to note that a fiducial mark on the steel was used as the point of reference for each image taken. This was done so that all images across all rotational speeds could be aligned exactly with one another, ensuring that the same population of particles was tracked at each rotational speed. Due to image rotation that was required to accomplish the alignment and in order to avoid the fiducial marker, only an area (approximately $1.4 \mathrm{~mm}$ $\mathrm{X} 1 \mathrm{~mm}$ ) on the center of each image was processed. The area provided a representative sample size as a few hundred particles were within the area for all the initial images (0 RPM images). In this way, the particle count was performed in an accurate and consistent manner. This experimental procedure was used to determine the residual adhering percentage (RAP) of the silica particles from four separate areas on the stainless steel to provide a statistically-valid representation of the particle adhesion. 


\subsubsection{Silica Particle Size Distribution}

The particle size distribution for the silica particles used in this study, based on volume equivalent sphere diameter, was obtained using laser diffraction (Malvern Mastersizer 2000) and is shown in Fig. 2. The median particle size, $d_{50}$, was $23.8 \mu \mathrm{m}$, while the tenth and ninetieth percentiles were $9.82 \mu \mathrm{m}$ and $52.2 \mu \mathrm{m}$. A lognormal distribution was fit to the measured size distribution of the silica particles. The fitted distribution had the parameters $\mu=3.197$ with a $95 \%$ confidence interval of $(3.184-$ $3.210)$ and $\sigma=0.664$ with a $95 \%$ confidence interval of $(0.654-0.673)$. All simulations and calculations involving particles used the lognormal description to represent the measured distribution.

\subsubsection{Approach for Determination of Size-Dependent Effective Hamaker Constant Distribution}

In order to determine an effective Hamaker constant distribution, the van der Waals adhesion force of model spherical particles against a flat substrate [38] was calculated using the well-established approximate relationship:

$$
F_{v d W}(D)=\frac{A_{e f f} R}{6 D^{2}}
$$

where $F_{v d W}(D)$ is the van der Waals adhesion force, $A_{\text {eff }}$ denotes the effective Hamaker constant of the system, $R$ is the radius of the particle, and $D$ represents the separation distance between the two surfaces. The simplicity of this relationship offers an appealing form for the capture of the complex effects of particle roughness and shape distributions. The calculated adhesion force (Eq. 2) was related to the radial 
force (Eq. 1) to calculate the rotational speed required to dislodge the particles from the substrate.

In order to model the observed RAP curve of the silica particles against the stainless steel, the centrifuge technique was simulated assuming that the powder could be considered to be comprised of smooth spherical particles and that the van der Waals force could be described using the approximate sphere-flat plate relationship in Eq. 2. Adhesion calculations were accomplished by first randomly selecting 1,000 particles from the lognormal fitted distribution of the silica powder. To maintain the same size range as the silica particle size distribution, limits of 2 and $105 \mu \mathrm{m}$ were placed on the lognormal distribution. If particles with diameters outside this range were selected for use in the simulation, these particles were rejected and new particles were selected until ones with diameters within the specified range were identified. The van der Waals adhesion force of each particle against the plates was then calculated using an arbitrarily-selected Hamaker constant of $5 \times 10^{-21} \mathrm{~J}$, the measured particle radius $(R)$, and a separation distance $(D)$ at contact of $0.4 \mathrm{~nm}$, a common value for the separation distance when two surfaces are in contact [39], in Eq. 2. The initial low value of the Hamaker constant was selected because, from a modeling perspective, starting with a relatively small value for the Hamaker constant and adjusting upward when determining the distribution of effective Hamaker constants is a straightforward process.

It was necessary to set the calculated adhesion force to the radial force in order to simulate the centrifuge experiment by using:

$$
F_{a d}=F_{c e n t}=m \omega^{2} r_{c}
$$


The mass of each particle was calculated using the volume of the particle (assuming spherical geometry and using its radius, $R$ ) and its density, $\rho_{p}$, so that Eq. 3 becomes:

$$
F_{a d}=\frac{4}{3} \pi R^{3} \rho_{p} \omega^{2} r_{c}
$$

For the simulations, both $\rho_{p}$ and $r_{c}$ were held constant, at $2.2 \mathrm{~g} / \mathrm{cm}^{3}$ and $9 \mathrm{~cm}$. The required rotational speed for the removal of each particle was calculated by solving for the angular velocity in Eq. 4 and converting it to revolutions per minute (RPM) by the expressions:

$$
\begin{gathered}
\omega=\sqrt{\frac{3 F_{a d}}{4 \pi R^{3} \rho_{p} r_{c}}} \\
R P M_{\text {removal }}=\frac{60 \omega}{2 \pi}
\end{gathered}
$$

With the removal RPM determined, the simulated RAP was generated. This was accomplished by determining the number of particles removed at set rotational speeds and then calculating the percentage of particles remaining as a function of those speeds. Centrifuge technique simulation results were compared to experimental results to determine values of the effective Hamaker constant that allowed the experimental data to be represented by a powder comprised of smooth spheres whose van der Waals adhesion force was described in terms of a size-dependent distribution of effective Hamaker constants that accounted for the particle roughness. This was accomplished by reconstructing the experimental RAP curve with simulation results. To reconstruct the experimentally observed RAP curve for the real particle removal from the surface in terms of the adhesion of idealized smooth spheres, the Hamaker constant in the smooth sphere adhesion description was adjusted, as a function of particle size. 
To achieve this outcome, the simulated removal data for the smooth particles was replotted as the required speed for removal (RPM removal) as a function of particle size.

A method was developed to use the re-plotted data to determine the effective Hamaker constants as follows:

1. The percent of particles removed experimentally was determined at the

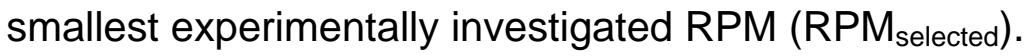

2. The original particle size distribution was consulted and a particle size was selected such that the percentage of particles larger than the identified size equaled the percentage of particles removed in step 1 (e.g., if $13 \%$ of the particles were removed, then $13 \%$ of the original distribution was larger than the selected particle size).

3. The Hamaker constant used to describe the van der Waals adhesion for the smooth particles smaller than the size from step 2 was adjusted so that the expected removal RPM of these particles was increased to be slightly above the RPM selected from step 1. This alteration brought the RAP for the simulated smooth particles into agreement with the experimentally observed RAP at the selected RPM.

4. The next RPM used experimentally was selected (i.e., if $1500,3000,4500$, and 6000 RPM were the rotational speeds investigated, then the first rotational speed considered in this scheme would be 1500 , followed sequentially by 3000,4500 , and then 6000 ), and was identified as the new $\mathrm{RPM}_{\text {selected. }}$

5. The percentage of particles removed at the new RPM $\mathrm{M}_{\text {selected }}$ was identified. 
6. The particle size corresponding to the percentage of particles removed in step 5 was identified.

7. Considering only the particles smaller in size than the size identified in step 6 , new Hamaker constants were calculated for these particles using the relationships of the adhesion force, angular velocity and removal RPM from Eqs. 2, 5, and 6 using a removal RPM that was slightly greater than the $\mathrm{RPM}_{\text {selected. In this manner, the percent of particles adhering at the RPM }} \mathrm{M}_{\text {selected }}$ in step 6 was attained.

8. Steps 4 through 7 were repeated for all remaining rotational speeds investigated experimentally. Each time, the upper limit for the particle size in the Hamaker constant tuning was the lower limit from the prior tuning step.

The percent of theoretical (smooth, spherical) particles adhering at each RPM (as calculated using the calculated Hamaker constant distribution) can now be plotted as a function of RPM to construct an improved theoretical RAP curve.

It is important to note that the results obtained by this technique are only specific to the system being studied, and the extracted parameters will be dependent on the materials involved and the processing conditions used to create them. Variations in the extracted parameters for multiple similarly-composed and similarly-processed systems have not been studied in this first-generation work, so it is not yet known if the fitted parameters extracted for one system can be used for others, or if they must be extracted uniquely for each system of interest. Also, the approach works best for particles that are in the $\sim 1-150 \mu \mathrm{m}$ range. Smaller particles are difficult to dislodge 
with the centrifuge and are harder to see with an optical microscope. Larger particles tend to be too massive and will be displaced by the centrifuge quite easily.

\section{Results and Discussion}

\subsection{Centrifuge Technique Experimental and Simulation Results}

Fig. 3 shows the particles still remaining on the stainless steel after the centrifuge was rotated at $0,3000,6000$, and 9000 RPM. By comparing the images, one can see that increasing the rotational speed of the centrifuge increases the radial force and the removal of the particles. The percentage of particles remaining for the different areas was calculated from the particle counts. Averages of the percent of particles remaining along with the standard deviations were plotted for each rotational speed, shown in Fig 4. Particle removal is gradual initially up to 3000 RPM as only the most massive particles are removed at these rotational speeds. The majority of removed particles are dislodged between 4500 and 9000 RPM, accounting for the particles at the center of the size distribution. After this point, only a small fraction of particles is removed at the highest rotational speed of 10500 RPM. Because of their low mass, these particles require very high rotational speeds before sufficient force can be exerted to remove them from the plates. The upper limit of operation for the centrifuge is roughly 10500 RPM. Approximately $40 \%$ of the particles remain adhered at this rotational speed. Higher rotational speeds, beyond the operational limit of the centrifuge used in this study, are required to remove these remaining particles. 
Eqs. 2, 5, and 6 were followed from Section 2.2.3, along with the values mentioned therein for the parameters in each equation, in order to simulate the RAP of an ideal system. As an example calculation, a $25 \mu \mathrm{m}$ diameter smooth particle has a calculated adhesion force of $65 \mathrm{nN}$ from Eq. 2 under this condition. The angular velocity that would produce a removal force of $65 \mathrm{nN}$ on this particle was calculated by plugging the $65 \mathrm{nN}$ adhesion force into Eq. 5, and was determined to be $200 \mathrm{rad} / \mathrm{s}$. This was then converted to RPM with Eq. 6, to attain a value of 1910 RPM. This means that the 25 $\mu \mathrm{m}$ diameter smooth particle will be removed once the rotational speed used in the simulation is greater than 1910 RPM. This was repeated for every randomly selected particle to simulate the centrifuge technique.

The simulated RAP for the ideal system with a singular Hamaker constant (hollow circles, dashed line) is also plotted in Fig. 4. As can be seen, this does not recreate the RAP found experimentally. Thus, in order to use smooth particles to reconstruct the experimental RAP curve, the adhesion force of the smooth particles had to be altered in some fashion. It should be noted that each data point in Fig. 4 is composed of several (in fact, many) adhesion forces for various particle sizes being removed at a particular rotational speed. Another note is that in order to perform these calculations, it was necessary to work from the pre-measured size distribution for the powder. The particle removal from the nominally flat steel does not generate information that can be used to determine the size distribution. In other work in which this method is executed using plates with highly-engineered topography, it is possible to extract the size distribution from the removal curves [34]. 


\subsection{Reconstruction of Experimental RAP Curve Using Ideal Particles with an Effective}

Hamaker Constant Distribution

If an appropriate Hamaker constant distribution were used to predict the adhesion forces of the idealized particles, the difference between the percent of particles remaining in the simulated and experimental RAP curves (Fig. 4) would be minimized. As described in Section 2.2.3, simulated results were re-plotted with the removal RPM presented as a function of particle size, as displayed in Fig. 5. In this plot, for any removal RPM of interest (RPM removal), a horizontal line intersecting the particle size divides the plot into two groups of particles: those remaining adhered at the rotational speed (rotational speeds and particle sizes above and to the left of the intersection), and those removed at that speed (rotational speeds and particle sizes below and to the right of the intersection), as shown in Fig. 6. This was the basis for the methodology described in Section 2.2.3 that was used to adjust the Hamaker constant. Fig. 7 shows the change in the RPM required to remove an ideal, smooth spherical powder with a singular Hamaker constant from the plates as a function of particle size, as well as the required RPM for this same powder if the Hamaker constant is adjusted so that the particle removal at each rotational speed matches that observed experimentally. The step-like form of the removal rotational speeds using the adjusted Hamaker constants reflects the fact that only a fraction of the full particle size distribution was used to 'tune' the Hamaker constant at each selected RPM.

The result of this scheme was a distribution of effective Hamaker constants, each tuned by fitting the predicted adhesion to the observed behavior for a population of particles in a size range within the distribution. These are effective Hamaker constants 
because they have been adjusted to account for the effects of the particle roughness on the adhesion. The size dependency results when the Hamaker constant for the original smooth RAP curve is modified based on the behavior of particles from different regions in the size distribution. Fig. 8 shows the range of calculated effective Hamaker constants for each rotational speed and shows that the data is composed of separate series due to the discrete nature of the RPM values used in the experiments.

A lognormal distribution as a function of particle diameter, $d_{p}$, was used as the functional form to describe the effective Hamaker constant distribution in Fig. 8:

$$
A_{e f f}\left(d_{p} ; \mu m\right)=\frac{\alpha}{\sigma \sqrt{2 \pi}} e^{\frac{-\left(\ln d_{p}-\mu\right)^{2}}{2 \sigma^{2}}}+\beta
$$

Fitting parameters $\alpha$ and $\beta$ were applied to the typical lognormal equation with its parameters $\mu$, the mean, and $\sigma$, the standard deviation. The resultant fit had an Rsquared value of 0.7929 with parameters: $\alpha=189.5 \times 10^{-21} \mathrm{~J}, \beta=-0.3413 \times 10^{-21} \mathrm{~J}$, $\mu=3.465$, and $\sigma=0.7417$. As can be seen in Fig. 8, the lognormal fit (smooth line) goes through the mean of each set of constants, except for the last set. It is hypothesized that the lognormal form of the Hamaker constant distribution is driven by the lognormal nature of the particle size distribution.

The improved theoretical RAP curve for the ideal smooth particles (obtained with the distribution of effective Hamaker constants following the method in Section 2.2.3) is presented with the observed RAP curve for the rough (real) powder in Fig. 9. As can be seen, the reconstruction of the experimental RAP curve in terms of smooth particles was accomplished successfully. Effective Hamaker constants calculated using Eq. 7, based on the parameters extracted from Fig. 8, were also used to calculate the 
theoretical particle removal at each particle size. Fig. 10 displays the improved theoretical RAP curve generated using the calculated effective Hamaker constants from Eq. 7 and the observed RAP curve obtained experimentally through the centrifuge technique. It is seen that the equation for the effective Hamaker constant distribution allows the adhesion behavior of the rough particles to be described in terms of ideal, smooth particles with fitted effective Hamaker constants.

\section{Conclusion}

The adhesion of silica particles to stainless steel was investigated via the centrifuge technique using a modified analytical scheme. Experimental results were typical of the technique as particles were removed with sequential increases in the rotational speed. Simulations of the technique were then employed in which the removal of the real particles was simulated in terms of the behavior of a system of ideal particles. By comparing the experimental and simulated data, a size-dependent effective Hamaker constant distribution that captured the effects of the particle roughness on the adhesion was identified. Through using this size-dependent effective Hamaker constant distribution, it was demonstrated that the van der Waals adhesion of a population of smooth particles of ideal shape and morphology can be used to describe the adhesion of a population of rough, nonuniform particles. The effective Hamaker constant distribution captures the effects of size, shape, and topography of the particles investigated. This proof-of-concept study was completed for only one system, and future work will investigate how the outcomes change with other particulate systems that introduce different properties (e.g., different composition and variations in shape, 
size, and density). In practice, this method allows the adhesion of a powder to be characterized using simple experiments and a simple computational scheme, which should be of great interest to practitioners involved with powder processing operations.

\section{Acknowledgements}

The authors acknowledge the support provided by the US National Science

Foundation through the Engineering Research Center for Structured Organic Particulate Systems under grant EEC-0540855.

\section{References}

[1] M. Landin, P. York, M.J. Cliff, R.C. Rowe, A.J. Wigmore, Scale-up of a pharmaceutical granulation in fixed bowl mixer-granulators, Int. J. Pharm. 133 (1996) 127-131. doi:10.1016/0378-5173(95)04427-2.

[2] N. Harnby, An engineering view of pharmaceutical powder mixing, Pharm. Sci. Technol. Today. 3 (2000) 303-309. doi:10.1016/S1461-5347(00)00283-2.

[3] J. Prescott, R. Barnum, On powder flowability, Pharm. Technol. 24 (2000) 60-84. citeulike-articleid:11996698\nhttp://cat.inist.fr/?aModele=afficheN\&cpsidt=795318.

[4] F.J. Muzzio, T. Shinbrot, B.J. Glasser, Powder technology in the pharmaceutical industry: The need to catch up fast, Powder Technol. 124 (2002) 1-7. doi:10.1016/S0032-5910(01)00482-X.

[5] T. Freeman, The importance of powder characterisation, Pharm. Technol. Eur. 22 (2010) 21-26.

[6] A. Rogers, M. lerapetritou, Challenges and opportunities in pharmaceutical manufacturing modeling and optimization, Comput. Aided Chem. Eng. 34 (2014) 144-149. doi:10.1016/B978-0-444-63433-7.50015-8.

[7] K. V. Gernaey, A.E. Cervera-Padrell, J.M. Woodley, A perspective on PSE in pharmaceutical process development and innovation, Comput. Chem. Eng. 42 (2012) 15-29. doi:10.1016/j.compchemeng.2012.02.022. 
[8] A. Fadeyibi, Z.D. Osunde, G. Agidi, E.C. Evans, Flow and strength properties of cassava and yam starch-glycerol composites essential in the design of handling equipment for granular solids, J. Food Eng. 129 (2014) 38-46.

doi:10.1016/j.jfoodeng.2014.01.006.

[9] J. Fitzpatrick, L. Ahrne, Food powder handling and processing: Industry problems, knowledge barriers and research opportunities, Chem. Eng. Process. 44 (2005) 209-214. doi:10.1016/j.cep.2004.03.014.

[10] J. Litster, Ennis Bryan, The Science and Engineering of Granulation Processes, Kluwer Academic Publishers, Dordrecht, 2004.

[11] F. Podczeck, Particle-particle Adhesion in Pharmaceutical Powder Handling, Imperial College Press, London, 1998.

[12] D.M. Schaefer, M. Carpenter, B. Gady, R. Reifenberger, L.P. Demejo, D.S. Rimai, Surface roughness and its influence on particle adhesion using atomic force techniques, J. Adhes. Sci. Technol. 9 (1995) 1049-1062. doi:10.1163/156856195X00897.

[13] K. Cooper, N. Ohler, A. Gupta, S.P. Beaudoin, Analysis of contact interactions between a rough deformable colloid and a smooth substrate, J. Colloid Interface Sci. 222 (2000) 63-74.

[14] Q. Li, V. Rudolph, W. Peukert, London-van der Waals adhesiveness of rough particles, Powder Technol. 161 (2006) 248-255.

doi:10.1016/j.powtec.2005.10.012.

[15] K.N.G. Fuller, D. Tabor, The effect of surface roughness on the adhesion of elastic solids, Proc. R. Soc. Lond. A. Math. Phys. Sci. 345 (1975) 327-342.

[16] K. Cooper, a Gupta, S. Beaudoin, Substrate Morphology and Particle Adhesion in Reacting Systems., J. Colloid Interface Sci. 228 (2000) 213-219. doi:10.1006/jcis.2000.6881.

[17] K. Cooper, A. Gupta, S. Beaudoin, Simulation of Particle Adhesion: Implications in Chemical Mechanical Polishing and Post Chemical Mechanical Polishing Cleaning, J. Electrochem. Soc. 148 (2001) G662. doi:10.1149/1.1409975.

[18] S. Eichenlaub, A. Gelb, S. Beaudoin, Roughness models for particle adhesion, J. Colloid Interface Sci. 280 (2004) 289-298. doi:10.1016/j.jcis.2004.08.017.

[19] L. Suresh, J. Walz, Effect of surface roughness on the interaction energy between a colloidal sphere and a flat plate, J. Colloid Interface Sci. 183 (1996) 199-213. http://www.sciencedirect.com/science/article/pii/S0021979796905354 (accessed December 11, 2012). 
[20] H. Rumpf, Particle Technolgy, Chapman and Hall, London: New York, 1990.

[21] Y. Rabinovich, J. Adler, a Ata, R. Singh, B. Moudgil, Adhesion between Nanoscale Rough Surfaces., J. Colloid Interface Sci. 232 (2000) 10-16. doi:10.1006/jcis.2000.7167.

[22] J.F. Archard, Elastic Deformation and the Laws of Friction, Proc. R. Soc. A Math. Phys. Eng. Sci. 243 (1957) 190-205. doi:10.1098/rspa.1957.0214.

[23] K. Komvopoulos, Surface engineering and microtribology for microeleetromechanical systems, Wear. 200 (1996) 305-327.

[24] K. Komvopoulos, W. Yan, Three-Dimensional Elastic-Plastic Fractal Analysis of Surface Adhesion in Microelectromechanical Systems, J. Tribol. 120 (1998) 808. doi:10.1115/1.2833783.

[25] R.L. Jackson, A model for the adhesion of multiscale rough surfaces in MEMS, Proc. Annu. Southeast. Symp. Syst. Theory. (2011) 257-262. doi:10.1109/SSST.2011.5753817.

[26] S. You, M.P. Wan, Mathematical models for the van der Waals force and capillary force between a rough particle and surface, Langmuir. 29 (2013) 9104-9117. doi:10.1021/la401516m.

[27] D.J. Quesnel, D.S. Rimai, Finite elemental modeling of particle adhesion: a surface energy formalism, J. Adhes. 74 (2000) 177-194. doi:10.1080/00218460008034529.

[28] J.A. Greenwood, J.B.P. Williamson, Contact of Nominally Flat Surfaces, Proc. R. Soc. A Math. Phys. Eng. Sci. 295 (1966) 300-319. doi:10.1098/rspa.1966.0242.

[29] H. Krupp, Particle adhesion theory and experiment, Adv. Colloid Interface Sci. 1 (1967) 111-239. doi:10.1016/0001-8686(67)80004-6.

[30] G. Boehme, H. Krupp, H. Rabenhorst, G. Sandstede, Adhesion measurements involving small particles, Trans. Inst. Chem. Eng. 40 (1962) 252-259.

[31] G. Donsi', S. Moser, L. Massimilla, Solid-solid interaction between particles of a fluid bed catalyst, Chem. Eng. Sci. 30 (1975) 1533-1535. doi:10.1016/00092509(75)85036-6.

[32] H. Mizes, Small particle adhesion: measurement and control, Colloids Surfaces A Physicochem. Eng. Asp. 165 (2000) 11-23. doi:10.1016/S0927-7757(99)00442-2.

[33] G.R. Salazar-Banda, M.A. Felicetti, J.A.S. Gonçalves, J.R. Coury, M.L. Aguiar, Determination of the adhesion force between particles and a flat surface, using 
the centrifuge technique, Powder Technol. 173 (2007) 107-117. doi:10.1016/j.powtec.2006.12.011.

[34] M.C. Thomas, S.P. Beaudoin, An Enhanced Centrifuge-Based Approach to Powder Characterization: Particle Size and Hamaker Constant Determination, Powder Technol. 286 (2015) 412-419. http://dx.doi.org/10.1016/j.powtec.2015.08.010.

[35] P. Thévenaz, U.E. Ruttimann, M. Unser, A pyramid approach to subpixel registration based on intensity, IEEE Trans. Image Process. 7 (1998) 27-41. doi:10.1109/83.650848.

[36] C.M. Costa, S. Yang, Counting pollen grains using readily available, free image processing and analysis software., Ann. Bot. 104 (2009) 1005-10. doi:10.1093/aob/mcp186.

[37] C.A. Schneider, W.S. Rasband, K.W. Eliceiri, NIH Image to ImageJ: 25 years of image analysis, Nat. Methods. 9 (2012) 671-675. doi:10.1038/nmeth.2089.

[38] S. Beaudoin, P. Jaiswal, A. Harrison, J. Laster, K. Smith, M. Sweat, et al., Fundamental forces in particle adhesion, in: K.L. Mittal, R. Jaiswal (Eds.), Part. Adhes. Remov., Scrivener, Beverly, MA, 2015: pp. 1-79.

[39] K. Cooper, A. Gupta, S. Beaudoin, Simulation of the Adhesion of Particles to Surfaces., J. Colloid Interface Sci. 234 (2001) 284-292. doi:10.1006/jcis.2000.7276.

Vitae

Myles C. Thomas received his B.S. in biological engineering from Utah State University (2010). He received his Ph.D. in chemical engineering at Purdue University in August 2015. His Ph.D. thesis is entitled "Enhanced Centrifuge-Based Approach to Powder Characterization."

Stephen P. (Steve) Beaudoin received his B.S., M.S., and Ph.D. degrees in chemical engineering from MIT (1988), UT-Austin (1990), and North Carolina State University (1995). He is a Professor and University Faculty Scholar at Purdue University. 
Beaudoin's research centers around the measurement and modeling of particle adhesion to various surfaces, with a specific focus on the behavior of rough particles and surfaces with non-uniform geometry. 


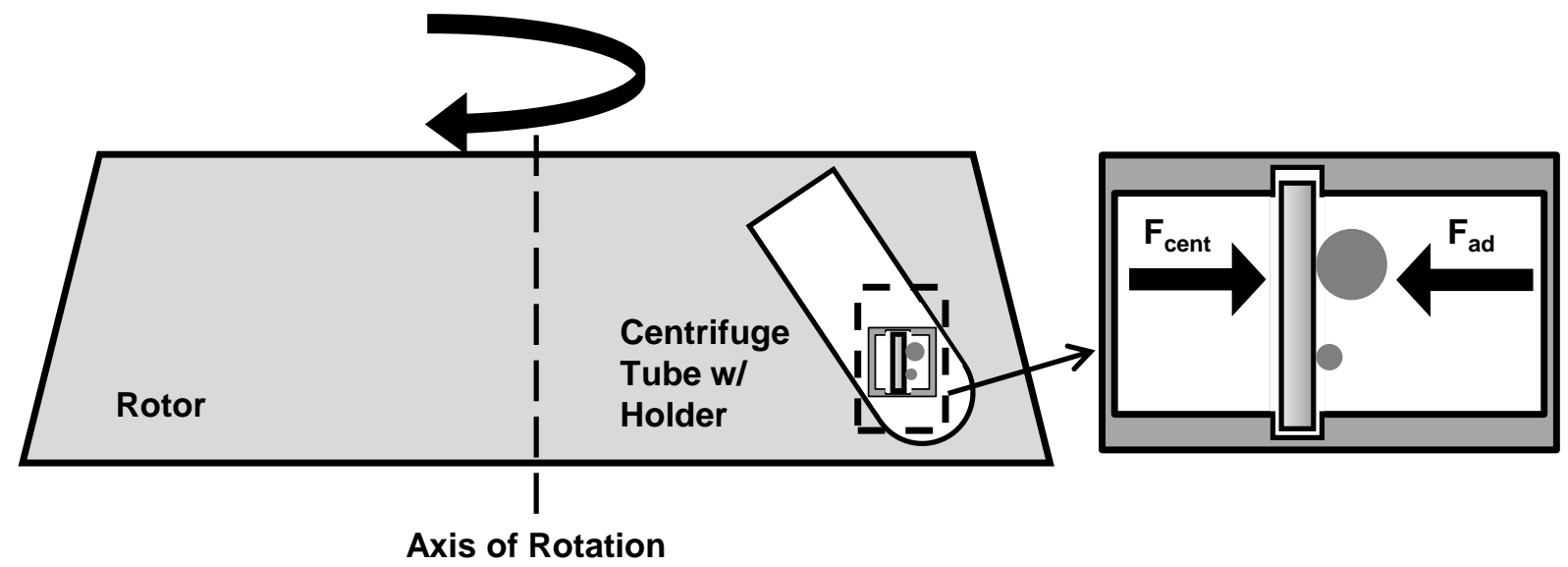




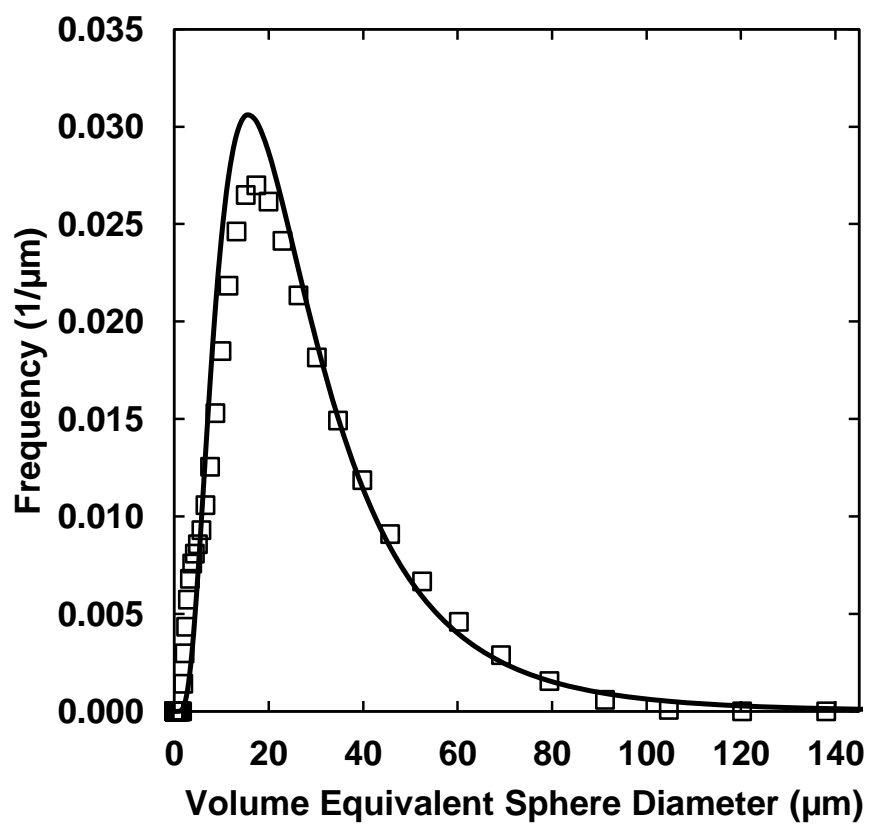



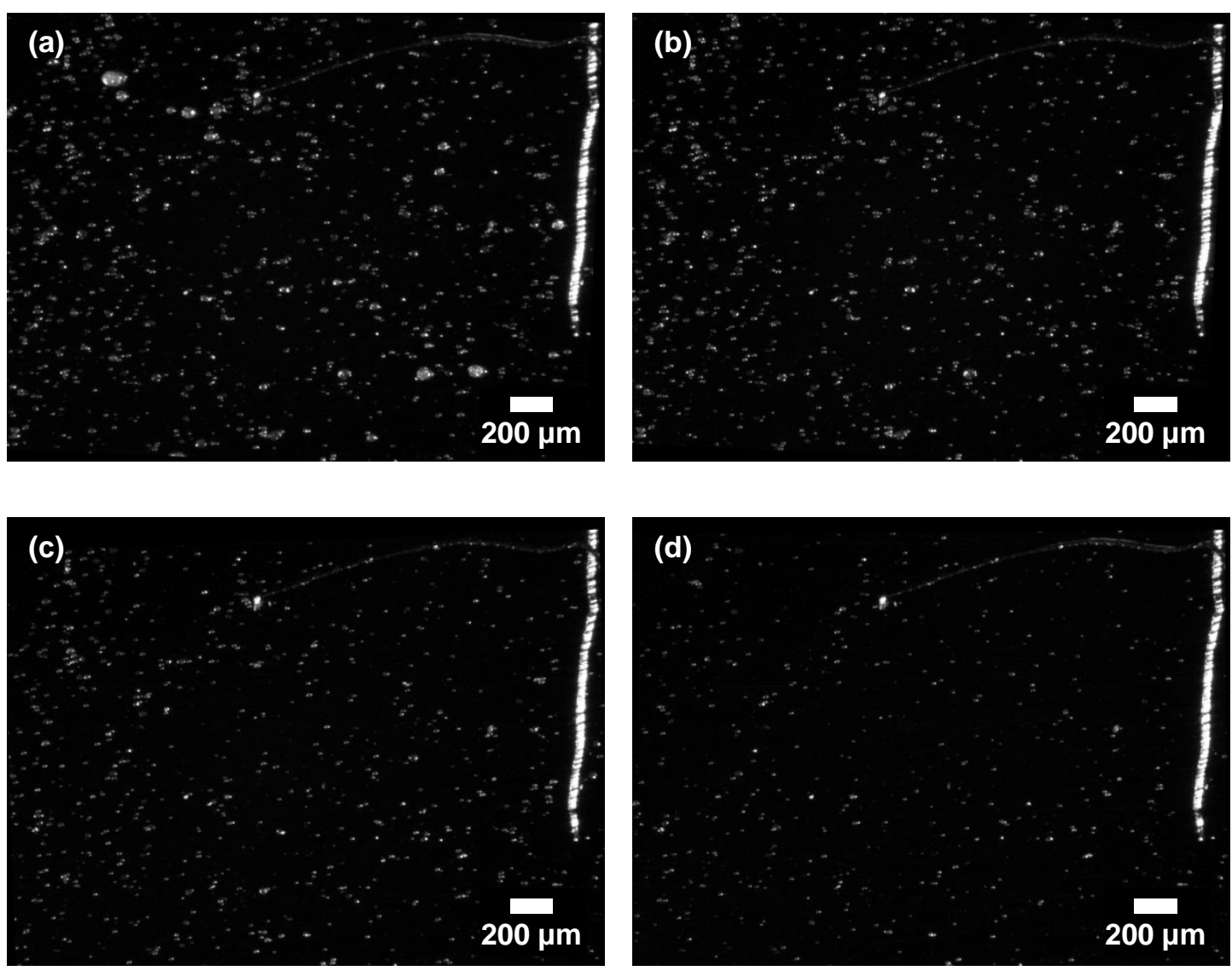


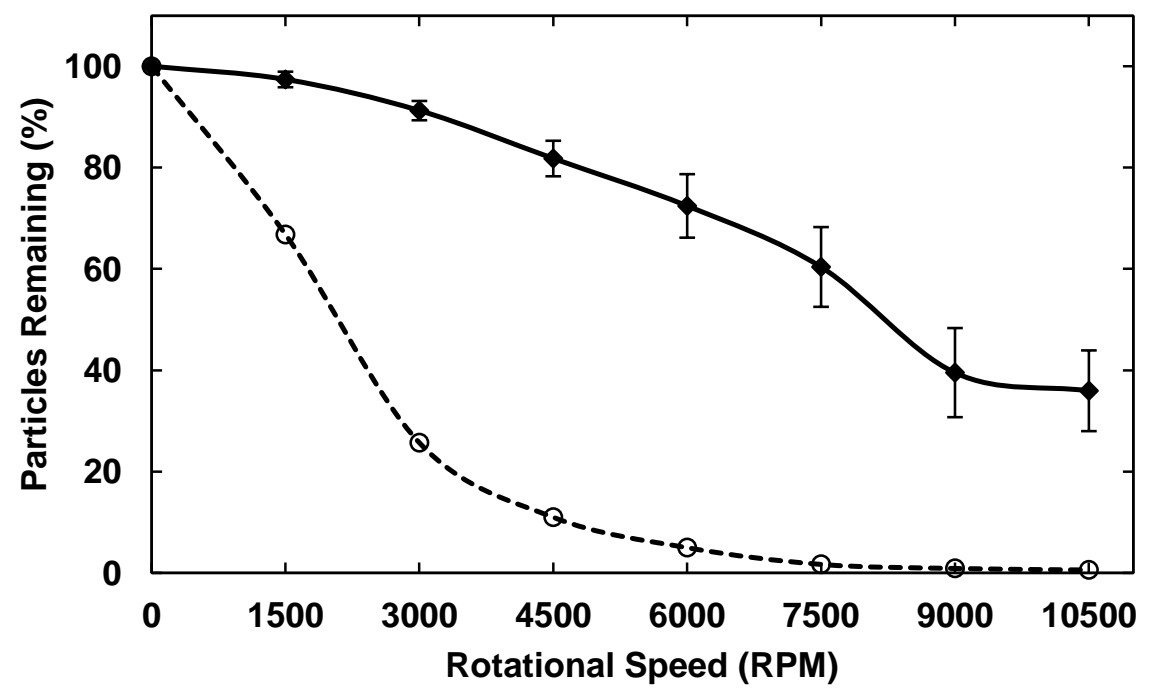




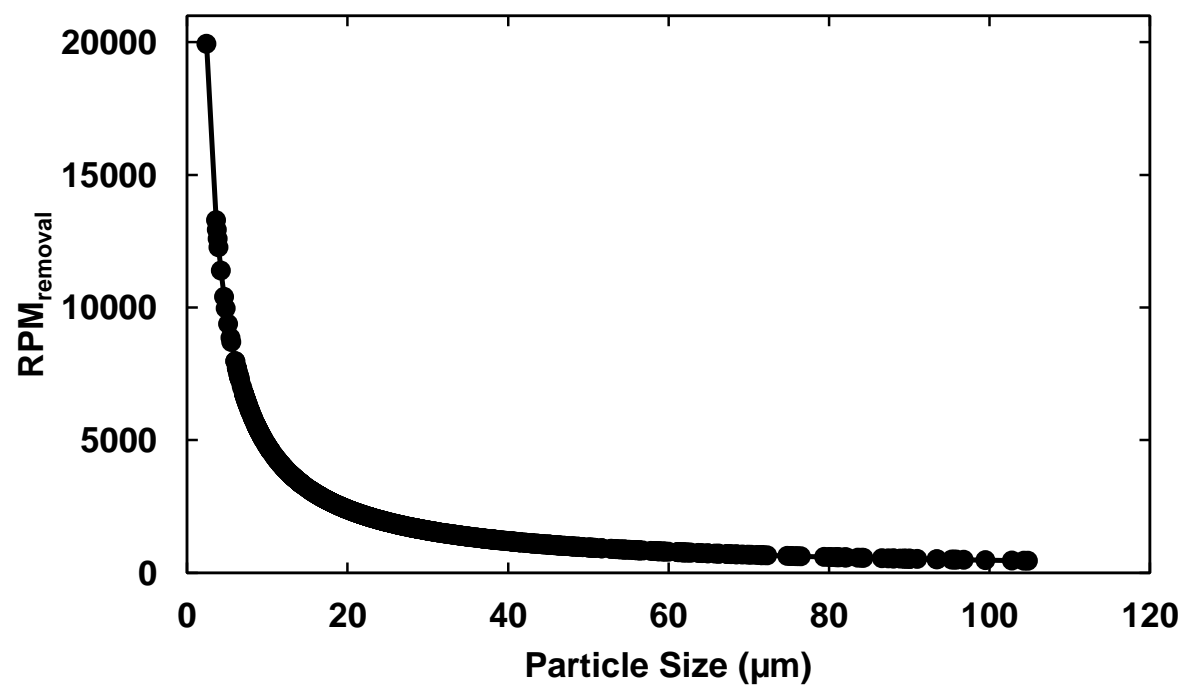




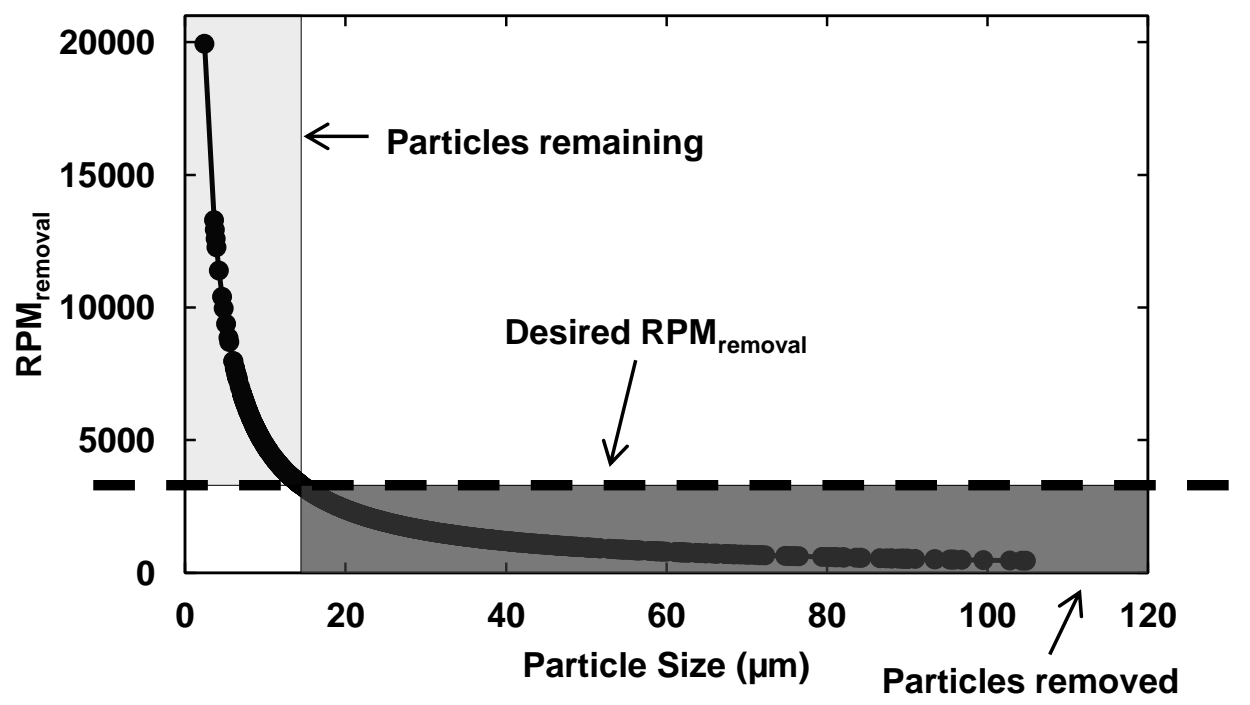




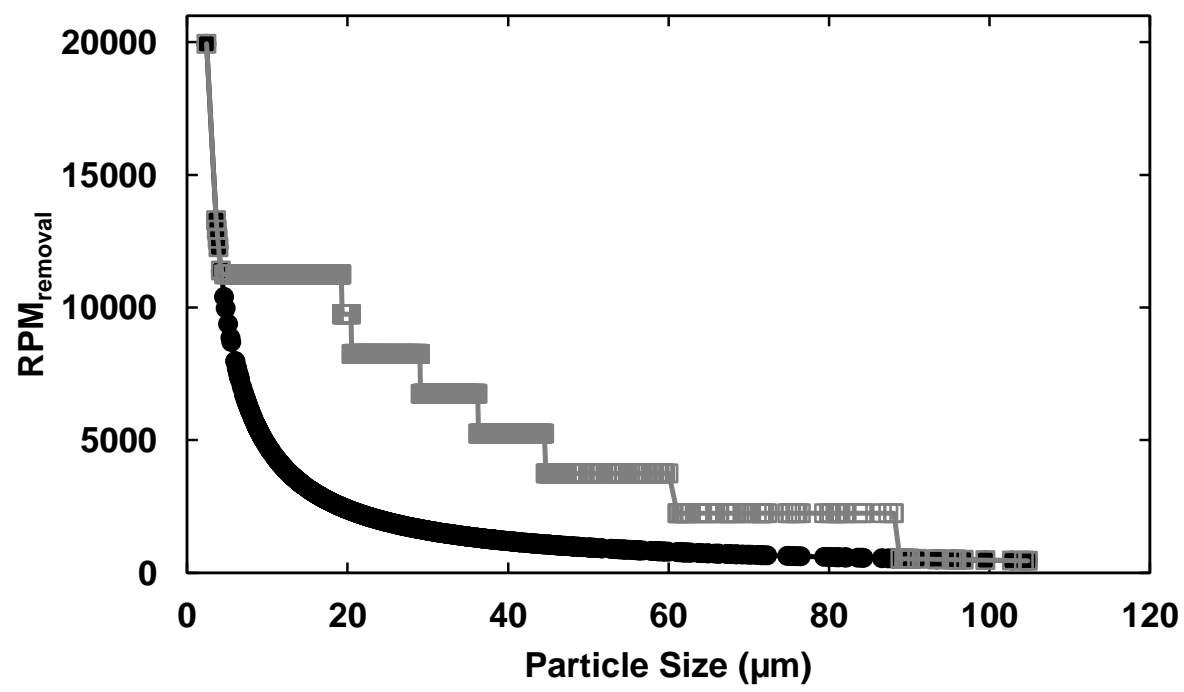




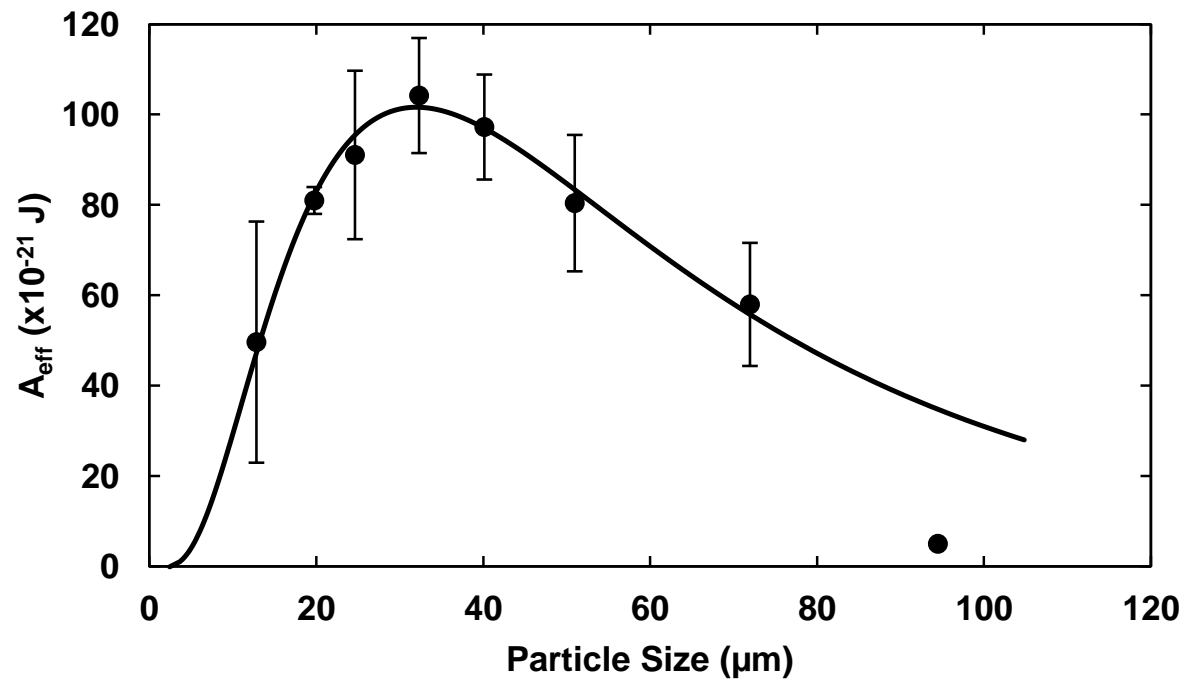


Figure(s)

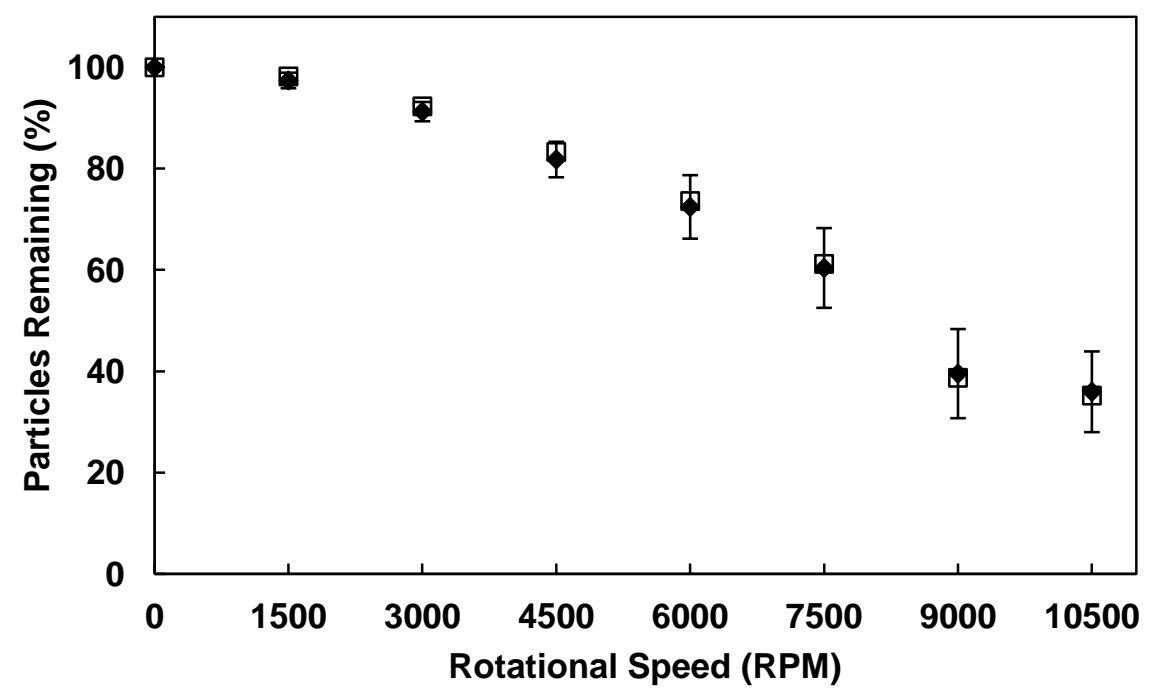


Figure(s)

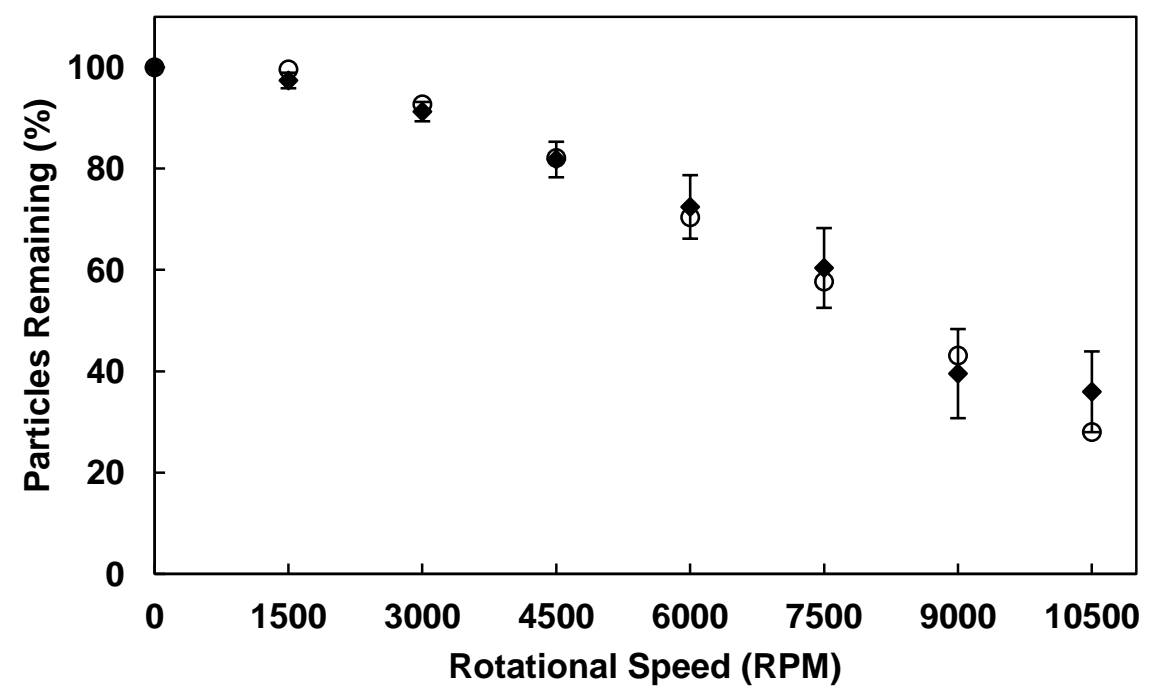


